\title{
La Respuesta Terapéutica a Ezetimiba en Ratones C57BL/6 es Mediada por Cambios en la Expresión de NPC1L1, ABCG5 y ABCG8 en el Enterocito
}

\author{
Therapeutic Response to Ezetimibe in C57BL/6 Mice is Mediated by Changes \\ in NPC1L1, ABCG5 and ABCG8 Expression in the Enterocyte \\ *,**,***** José M. Caamaño; ${ }^{*, * *}$ Nicolás Saavedra; ${ }^{* *}$ Cristian Wulff \& ${ }^{*, * *}$ Luis A. Salazar
}

CAAMAÑO, J.M.; SAAVEDRA, N.; WULFF, C. \& SALAZAR, L.A. La respuesta terapéutica a ezetimiba en ratones C57BL/6 es mediada por cambios en la expresión de NPC1L1, ABCG5 y ABCG8 en el enterocito. Int. J. Morphol., 30(2):531-540, 2012.

RESUMEN: Las proteínas NPC1L1, ABCG5 y ABCG8 participan en la absorción intestinal de colesterol. Ezetimiba inhibe este proceso bloqueando a NPC1L1, sin embargo, su efecto sobre ABCG5 y ABCG8 aún no está claro. Así, el objetivo del presente trabajo fue evaluar en ratones C57BL/6 con hipercolesterolemia inducida por dieta y tratados con ezetimiba, la expresión de NPC1L1, ABCG5 y ABCG8 mediante PCR en tiempo real y Western blot, en 3 grupos de animales: 1, dieta hipercolesterolémica D12336; 2, dieta D12336 más $5 \mathrm{mg} / \mathrm{kg} /$ día de ezetimiba; 3, dieta control. El nivel sérico de colesterol total fue significativamente diferente entre los grupos estudiados (control: 1,85 \pm 0,49 mmol/L; dieta D12336: 3,11 \pm 0,73 mmol/L; ezetimiba: 2,11 \pm 0,50 mmol/L, P=0,001). La expresión génica de NPC1L1 aumentó 5,4 veces en el grupo que recibió la dieta D12336 (P=0,003). Por otro lado, la expresión génica de ABCG5 y ABCG8 no fue diferente en el grupo con hipercolesterolemia $(\mathrm{P}=0,239$ y $\mathrm{P}=0,201$, respectivamente). Después del tratamiento con ezetimiba, la expresión génica de ABCG5 se incrementó 15,6 veces $(\mathrm{P}=0.038)$. No hubo diferencias significativas en la expresión génica de NPC1L1 $(\mathrm{P}=0,134)$ y ABCG8 $(\mathrm{P}=0,067)$. En relación a la expresión proteica, la dieta $\mathrm{D} 12336$ incrementó los niveles de expresión de NPC1L1 $(\mathrm{P}=0,022)$ y ABCG5 $(\mathrm{P}=0,008)$; el tratamiento con ezetimiba incrementó los niveles de NPC1L1 $(\mathrm{P}=0,048)$ y redujo los niveles de ABCG5 $(\mathrm{P}=0,036)$ y ABCG8 $(\mathrm{P}=0,016)$. En conclusión, nuestros resultados sugieren que tanto la dieta hipercolesterolémica como el tratamiento con ezetimiba, en un modelo experimental, afectan los niveles de expresión de NPC1L1, ABCG5 y ABCG8, sugiriendo que ABCG5 y ABCG8 están involucrados en la respuesta hipolipemiante a este fármaco. No obstante, el mecanismo mediante el cual se explica esta interacción requiere de un futuro estudio.

PALABRAS CLAVE: Ezetimiba; Colesterol; NPC1L1; ABCG5/G8.

\section{INTRODUCCIÓN}

Se ha establecido que la captación de colesterol por los enterocitos se debe a mecanismos facilitados por proteínas (Lammert \& Wang, 2005). En el año 2004, la proteína Niemann-Pick C1-like 1 (NPC1L1), fue identificada como responsable de la absorción de colesterol de la dieta a nivel intestinal (Altmann et al., 2004). Además, la cantidad de colesterol absorbido puede ser regulada a través de transportadores ATP-binding cassette (ABC), localizados en la membrana del enterocito, que ejercen su acción limitadora de absorción de colesterol mediante la secreción de vuelta al lumen intestinal del colesterol internalizado (Hui \&
Howles, 2005). ABCG5 y ABCG8, forman un heterodímero que está involucrado en el eflujo de esteroles de la dieta desde las células epiteliales intestinales hacia el lumen del intestino y del hígado al ducto biliar (Schmitz et al., 2001). Experimentos desarrollados en ratones knock-out para ABCG5/ABCG8 han permitido confirmar que ABCG5 y ABCG8 son los mayores transportadores hepatobiliares de colesterol, que protegen frente a la acumulación de esterol dietético en el cuerpo y que la estimulación de la excreción de colesterol en la bilis por agonistas de LXR requiere de ABCG5 y ABCG8 (Acalovschi et al., 2006).

\footnotetext{
" Centro de Biología Molecular \& Farmacogenética, Depto. de Ciencias Básicas, Facultad de Medicina, Universidad de La Frontera, Temuco, Chile.

** Núcleo de Desarrollo Científico - Tecnológico en Biorecursos, Universidad de La Frontera (BIOREN-UFRO), Temuco, Chile.

*** Departamento de Medicina Interna, Facultad de Medicina, Universidad de La Frontera, Temuco, Chile.

Este estudio fue financiado mediante Proyecto DIUFRO DI09-1007.
} 
Ezetimiba es un fármaco que previene el transporte de colesterol de la dieta y biliar a través de la pared intestinal sin afectar la absorción de vitaminas solubles en grasas, triglicéridos y ácidos biliares (Kosoglou et al., 2005). Ezetimiba tiene como blanco de acción la proteína NPC1L1 en la membrana del enterocito (Garcia-Calvo et al., 2005), y además, puede interaccionar con proteínas NPC1L1 hepáticas, afectando los niveles circulantes de colesterol (Temel \& Rudel, 2007), sin embargo, su mecanismo de acción aún no ha sido completamente establecido.

Ge et al. (2008), demostraron que la depleción de colesterol causa el transporte de NPC1L1 desde el compartimento de reciclaje endocítico hacia la membrana plasmática, mientras que el incremento de colesterol resulta en la internalización de NPC1L1. La endocitosis de NPCL1 requiere de microfilamentos y la unión al complejo clatrina/ AP2, de tal manera que la incorporación de colesterol a la célula intestinal estaría mediada por endocitosis vesicular. Por lo tanto, la ezetimiba ejercería su efecto de inhibición de la internalización de colesterol, impidiendo la unión de NPC1L1 al complejo clatrina/AP2 lo que finalmente, genera el bloqueo de la endocitosis de NPC1L1 (Ge et al.). Además, se ha demostrado que la ezetimiba es eficaz en el tratamiento de la sitosterolemia, enfermedad caracterizada por un estado de hiperabsorción de esteroles de plantas en el intestino delgado y que es causada por mutaciones en los genes ABCG5 y ABCG8 (Klett \& Patel, 2004). Adicionalmente, se ha descrito que la expresión de ARN mensajero (ARNm) de ABCG5 y ABCG8 en el intestino de pacientes tratados con estatinas es incrementada, lo que se encuentra relacionado con la limitación en la absorción de colesterol (Lally et al., 2006). Para evaluar de qué manera el tratamiento con ezetimiba puede afectar la expresión de NPC1L1, ABCG5 y ABCG8, se estudió la expresión de estos genes y proteínas en el intestino delgado de ratones C57BL/6, sometidos a una dieta rica en colesterol y posterior administración de ezetimiba.

\section{MATERIAL Y MÉTODOS}

Animales y diseño experimental. 15 ratones machos C57BL/6 fueron divididos en grupos de cinco, sometidos a 15 días de adaptación con ciclos de 12 horas de luz y oscuridad y alimentados a libre acceso con la dieta rata de laboratorio (Champion S.A.). Luego, a cinco de los animales se les extrajo sangre para medir los lípidos plasmáticos y extirpó el intestino delgado, para evaluar la expresión basal génica y proteica de NPC1L1, ABCG5 y ABCG8 (Duan et al., 2006). A los 10 animales restantes se les indujo hipercolesterolemia, alimentándolos por 4 semanas, a libre acceso con la dieta tipo Paigen; D12336 (Paigen et al., 1985). Luego, se les extrajo sangre para medir los lípidos plasmáticos. 5 ratones, denominados "tratados" continuaron consumiendo la dieta D12336 y recibieron mediante gavage $5 \mathrm{mg} / \mathrm{kg} /$ día de ezetimiba (Zient*, Schering-Plough), durante 30 días (Oswald et al., 2006). Los 5 restantes, fueron denominados "no tratados" y mantenidos en las mismas condiciones que los "tratados" pero sin ezetimiba. Finalmente, a los dos grupos se les extrajo sangre para las determinaciones de los niveles de lípidos y extirpo el intestino delgado, el que se almacenó a $-80^{\circ} \mathrm{C}$. El protocolo de manejo de estos animales se rigió por el Manual de Normas de Bioseguridad del Comité Nacional de Biotecnología de CONICYT (2008).

Determinación de los niveles séricos de lípidos. Las concentraciones séricas de colesterol total y triglicéridos se determinaron mediante métodos enzimático-colorimétricos (Fossati \& Prencipe, 1982; Fossati \& Medicci, 1987).

Evaluación de la expresión génica de NPC1L1, ABCG5 y ABCG8. El ARN total de los enterocitos de los ratones C57BL/6 se extrajo utilizando el reactivo comercial TRIZOL® (Invitrogen, Life Technologies, EE.UU.). La transcripción reversa para la síntesis de ADNc, se efectuó a partir de las muestras de ARN tratado con DNasa I. El análisis de la expresión de los genes ABCG5, ABCG8 y NPC1L1 fue realizado mediante reacción en cadena de la polimerasa en tiempo real, empleando las siguientes condiciones: 0,5 $\mu \mathrm{L}$ de cada primer $(200 \mathrm{nM}), 10 \mu \mathrm{L}$ de SYBR@ Green PCR Master Mix (Applied Biosystems, EE.UU.), $1 \mu \mathrm{L}$ de ADN complementario (ADNc) y agua desionizada estéril para un volumen final de $20 \mathrm{~mL}$. A continuación, los tubos fueron colocados en un termociclador 7300 Real Time PCR System (Applied Biosystems, EE.UU.) y sometidos al siguiente esquema de temperaturas: denaturación inicial a $95^{\circ} \mathrm{C}$ por 10 min., seguido de 45 ciclos a $95^{\circ} \mathrm{C}$ por 15 segundos (Denaturación), $57^{\circ} \mathrm{C}$ por 30 segundos (Hibridación), $72^{\circ} \mathrm{C}$ por 30 segundos (Extensión). Para determinar el nivel de expresión relativa se utilizó el método de Livak \& Schmittgen (2001), mediante el software Relative Expression Software tool (RESTC). La expresión de los genes fue normalizada en función de los niveles de ARN mensajero (ARNm) del gen GAPDH.

Análisis de la expresión proteica mediante Western blot. Para la extracción de proteínas, $5 \mathrm{mg}$ de yeyuno fueron pulverizados y homogeneizados empleando $300 \mathrm{~mL}$ de buffer RIPA al cual se adicionó cocktail inhibidor de proteasas (Haltä Protease Inhibitor Cocktail Kit, Pierce Chemical Co) y posteriormente, se centrifugó a $12.000 \mathrm{rpm}$ a $4^{\circ} \mathrm{C}$. En el sobrenadante se cuantificaron las proteínas con el kit BCAä Protein Assay (Pierce Chemical Co.). Las proteínas fueron 
separadas mediante electroforesis en gel denaturante de poliacrilamida con dodecil sulfato de sodio (SDS-PAGE) y transferidas a membrana Polivinilidenofluoruro (PVDF, Pierce Chemical Co.). La membrana fue saturada con solución bloqueadora (leche descremada al $5 \%$ en buffer TTBS $1 \mathrm{X})$ a $4{ }^{\circ} \mathrm{C}$. La reacción tuvo lugar por la adición de anticuerpos primarios anti-NPC1L1 (NPC1L1 (M-220): sc67237), anti-ABCG5 (ABCG5 (H-300): sc-25796) y antiABCG8 (ABCG8 (H-300): sc-30111) (Santa Cruz Biotechnology Inc., EE.UU.) y fue revelada con anticuerpo policlonal de conejo anti-IgG acoplado a peroxidasa de rábano (Cell Signaling Technology, Inc., EE.UU.). Se empleó un anticuerpo Anti-b-actina (Cell Signaling Technology, Inc., EE.UU.) como control de expresión. Para la visualización de las bandas se utilizó reactivo quimioluminiscente (Pierce Chemical, Rockford, EE.UU.). La película fue escaneada y almacenada para su posterior interpretación.

Análisis estadístico. Los resultados obtenidos fueron analizados mediante el programa SigmaPlot v. 11.0 (Systat Software Inc., CA, EE.UU.). Inicialmente, todas las variables se analizaron descriptivamente. Para las variables continuas, el análisis se realizó mediante la observación de valores mínimos y máximos, cálculo de promedios y desviaciones estándar.

La comparación entre las variables continuas se efectuó con los test t de Student o ANOVA para muestras pareadas. Además, se usó el método de Bonferroni para controlar la significancia estadística al hacer comparaciones múltiples. La comparación entre proporciones se evaluó por el test de Chi-cuadrado o test exacto de Fisher. El nivel de significancia considerado fue de $\mathrm{P}<0.05$.

\section{RESULTADOS}

Determinaciones bioquímicas. En la Tabla I se muestran los valores plasmáticos de colesterol total y triglicéridos en los ratones C57BL/6, al momento de ser incluidos en el estudio (valores basales) y después de consumir la dieta rica en colesterol D12336 durante 1 mes. Los animales que consumieron la dieta D12336 presentaron un incremento signi- ficativo $(P=0,01)$ en las concentraciones de colesterol total $(3,11 \pm 0,73 \mathrm{mmol} / \mathrm{L})$ comparados con el grupo basal $(1,85$ $\pm 0,49 \mathrm{mmol} / \mathrm{L})$. Por otra parte, a diferencia de lo observado con el colesterol total, los niveles de triglicéridos no presentaron un incremento significativo con el consumo de la dieta D12336 (1,08 $\pm 0,50$ vs. $0,73 \pm 0,30 \mathrm{mmol} / \mathrm{L}, \mathrm{P}=0,221)$.

El tratamiento con ezetimiba $(5 \mathrm{mg} / \mathrm{Kg} /$ día $)$ durante 4 semanas, demostró ser efectivo en la reducción de la hipercolesterolemia en los animales estudiados. El nivel de colesterol total fue significativamente más bajo en el grupo de animales tratado con ezetimiba comparado con el que consumió la dieta D12336, pero sin recibir el medicamento ( $3,11 \pm 0,73$ vs $2,11 \pm 0,50 ; P=0,002$, Tabla II), obteniéndose una reducción de un $32 \%$. No se observaron diferencias significativas para los valores plasmáticos de triglicéridos $(\mathrm{P}=$ $0,162)$.

Efecto de la hipercolesterolemia en la expresión relativa de los genes NPC1L1, ABCG5 y ABCG8 en células del intestino delgado de ratones C57BL/6. El análisis REST mostró que el gen NPC1L1 fue sobre-expresado 5,418 veces $(\mathrm{P}=0,003$; Fig.a 1A) en el grupo de animales que recibió la dieta D12336 en comparación con el grupo que fue alimentado con la dieta Champion (Grupo control). La expresión génica de ABCG5 y ABCG8 no presentó variación significativa en el grupo alimentado con dieta D12336 en relación al grupo control $(\mathrm{P}=0,239$ y $\mathrm{P}=0,201$, respectivamente).

Efecto del tratamiento con ezetimiba en la expresión relativa de los genes NPC1L1, ABCG5 y ABCG8 en células del intestino delgado de ratones $\mathrm{C} 57 \mathrm{BL} / 6$. El tratamiento con ezetimiba $(5 \mathrm{mg} / \mathrm{kg} /$ día $)$ durante 1 mes en los animales que desarrollaron hipercolesterolemia no mostró tener un efecto en la expresión del gen NPC1L1 comparado con el grupo basal $(\mathrm{P}=0,134)$. Por el contrario, el gen ABCG5 fue sobre-expresado en el grupo de animales tratado con ezetimiba con un aumento en los niveles relativos de ARNm de 15,6 veces $(\mathrm{P}=0,038$, Figura 1B) comparado con el grupo control. En el caso del gen ABCG8, si bien, es posible, apreciar una tendencia hacia la sobreexpresión, el análisis estadístico no muestra diferencia significativa $(\mathrm{P}=$ 0,067).

Tabla I. Concentraciones plasmáticas de lípidos antes y después del consumo de la dieta hipercolesterolémica D12336 en ratones $\mathrm{C} 57 \mathrm{BL} / 6$.

\begin{tabular}{lcccc}
\hline & Basal & Dieta D12336 & $\boldsymbol{P}^{*}$ & Valor referencia $^{* *}$ \\
\hline Colesterol Total (mmol/L) & $1,85 \pm 0,49$ & $3,11 \pm 0,73$ & 0,010 & $1,5-3,0$ \\
Triglicéridos (mmol/L) & $0,73 \pm 0,30$ & $1,08 \pm 0,50$ & 0,221 & $0,4-1,8$
\end{tabular}

*Test t de Student; ** Boehm et al. (2007). 
Tabla II. Concentraciones plasmáticas de lípidos en ratones C57BL/6 que consumieron dieta D12336 y después del tratamiento con ezetimiba $(5 \mathrm{mg} / \mathrm{kg} / \mathrm{día} / 1 \mathrm{mes})$.

\begin{tabular}{lcccc} 
& Dieta D12336 & TratamientoEzetimiba & $\boldsymbol{P}^{*}$ & Valor de referencia ** \\
\hline Colesterol Total $(\mathbf{m m o l} / \mathbf{L})$ & $3,11 \pm 0,73$ & $2,11 \pm 0,50$ & 0,002 & $1,5-3,0$ \\
Triglicéridos $(\mathbf{m m o l} / \mathbf{L})$ & $1,08 \pm 0,50$ & $0,78 \pm 0,32$ & 0,162 & $0,4-1,8$ \\
\hline
\end{tabular}

*Test t de Student; ** Boehm et al. (2007).
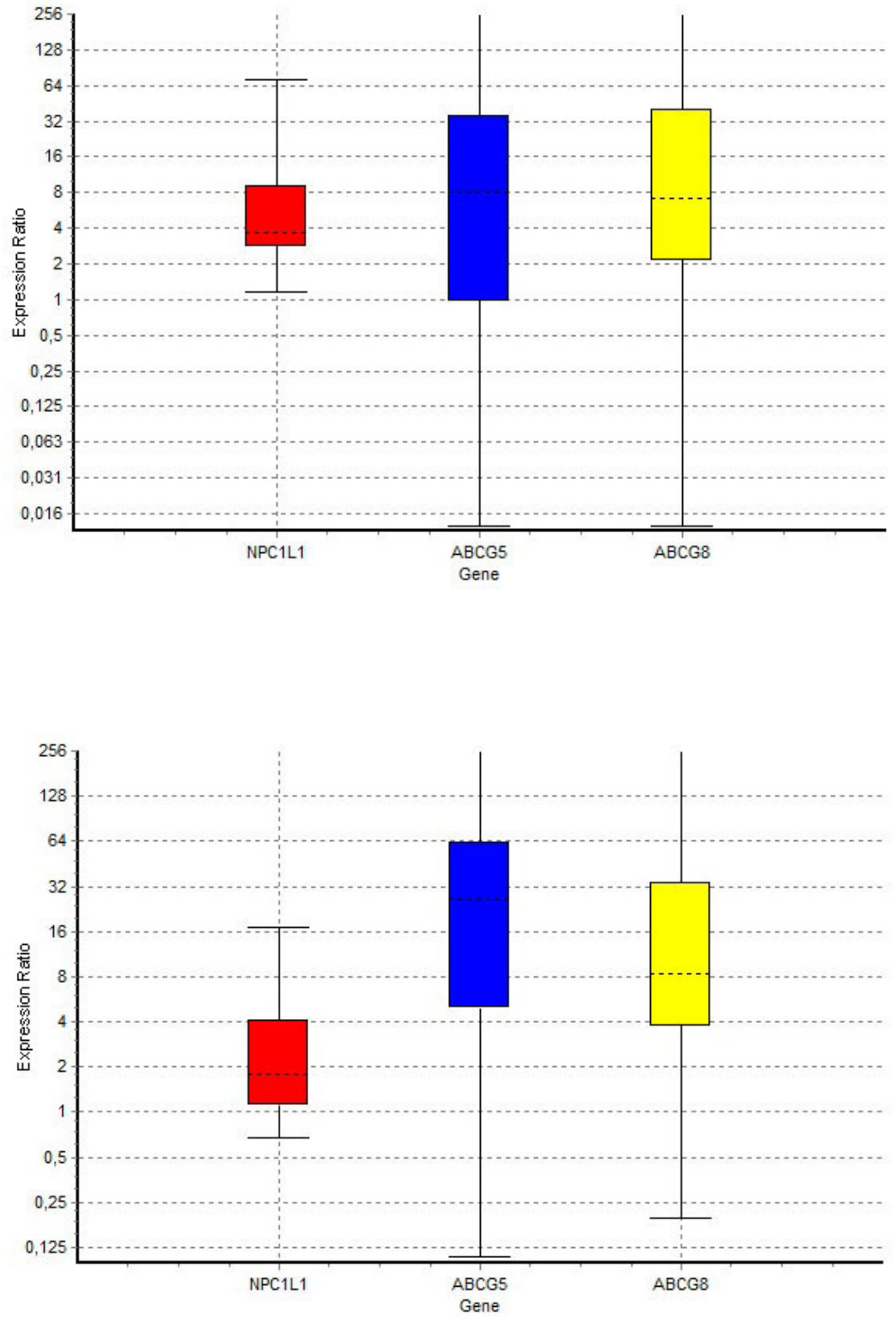

Fig. 1. Expresión relativa de los genes NPC1L1, ABCG5 y ABCG8 en células del intestino delgado de ratones C57BL/6 con hipercolesterolemia inducida por dieta D12336 (A) y tratados con ezetimiba (B), utilizando el Software REST 2008. Las determinaciones fueron realizadas por triplicado para cada animal.
Expresión de las proteínas NPC1L1, ABCG5 y ABCG8 en células del intestino delgado de ratones $\mathrm{C} 57 \mathrm{BL} / 6$ con hipercolesterolemia inducida por dieta. La Figura 2A ilustra los resultados del Western blot efectuado en las muestras de intestino delgado de los ratones C57BL/ 6 , después de ser alimentados durante un mes con la dieta rica en colesterol D12336, lo que provocó un aumento en la expresión intestinal de la proteína NPC1L1 comparado con los valores de expresión proteica basal $(0,368 \pm 0,14$ vs. $0,145 \pm$ 0,04; P = 0,022; Fig. 2B). Similarmente, para la proteína ABCG5, los valores de expresión se vieron alterados como resultado del consumo de la dieta rica en colesterol $(0,240 \pm 0,11$ vs. $2,26 \pm 1,48$; $\mathrm{P}=0,008)$. Por el contrario, la expresión de ABCG8 no presentó diferencias significativas $(1,19 \pm 0,07$ vs. $1,14 \pm 0,17 ; \mathrm{P}=$ $0,690)$.

Expresión de las proteínas NPC1L1, ABCG5 y ABCG8 en células del intestino delgado de ratones $\mathrm{C} 57 \mathrm{BL} / 6$ con hipercolesterolemia tratados con ezetimiba. La Figura 3A muestra los resultados del Western blot efectuado a partir de las muestras de intestino delgado de los ratones C57BL/6 con hipercolesterolemia inducida por dieta, después de ser tratados durante 1 mes con $5 \mathrm{mg} / \mathrm{kg} /$ día de ezetimiba. Al evaluar el efecto del tratamiento con ezetimiba (Figura 3B), se observaron diferencias significativas en el nivel de expresión de la proteína NPC1L1 $(0,368 \pm 0,14$ vs. 0,638 $\pm 0,19 ; \mathrm{P}=0,048)$. Así mismo, la administración de ezetimiba generó una diferencia en los niveles de expresión de las proteínas ABCG5 (2,26 $\pm 1,48$ vs. 0,132 $\pm 0,05 ; \mathrm{P}=0.036)$ y ABCG $8(1,143 \pm 0,165$ vs. $0,71 \pm 0,07 ; \mathrm{P}=0,016$ ). 

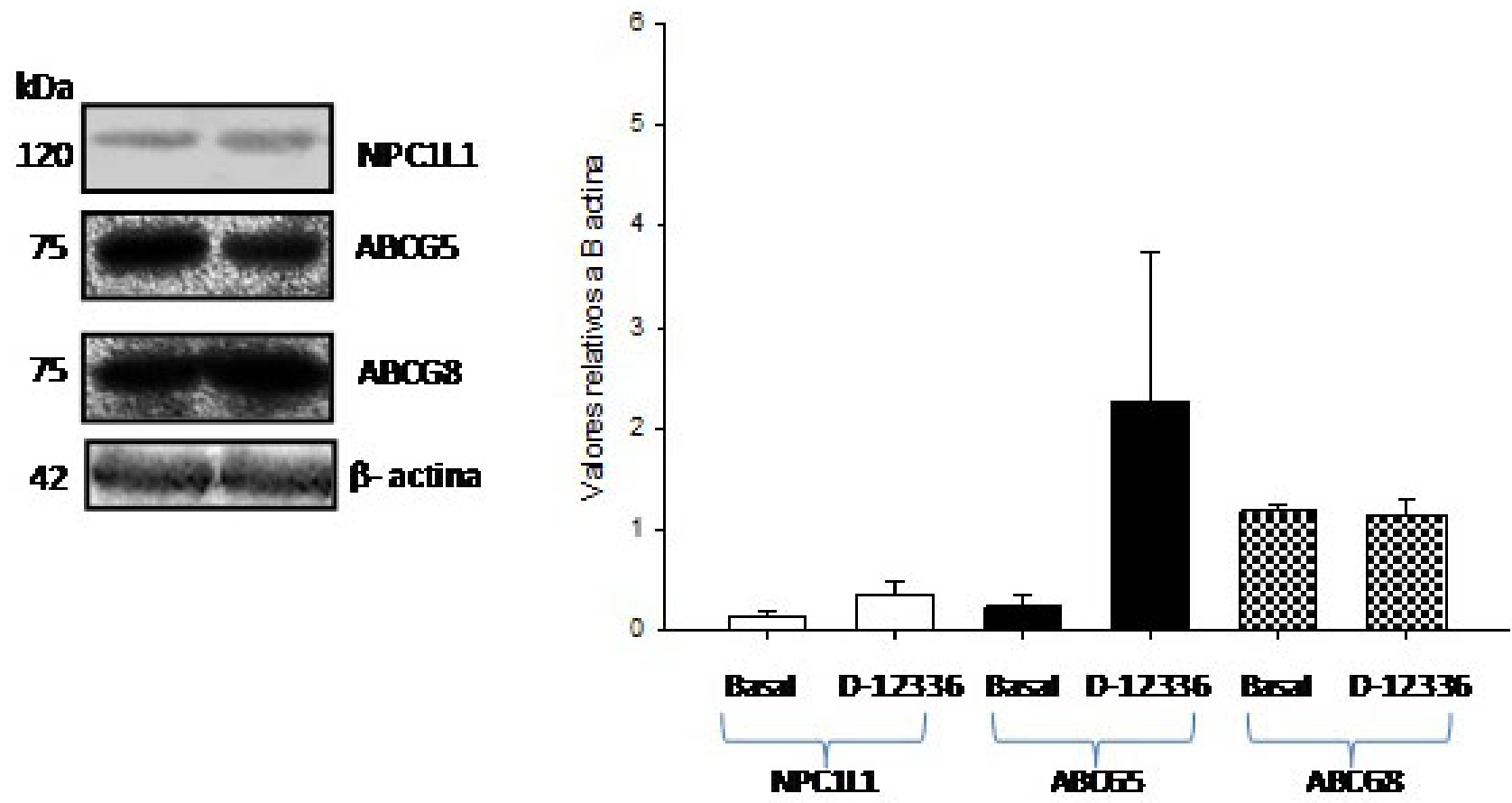

Fig. 2. Niveles de expresión de las proteínas NPC1L1, ABCG5 y ABCG8 en células del intestino delgado de ratones C57BL/6 con hipercolesterolemia, inducida por dieta D-12336. Los valores de expresión corresponden al promedio de 5 animales.
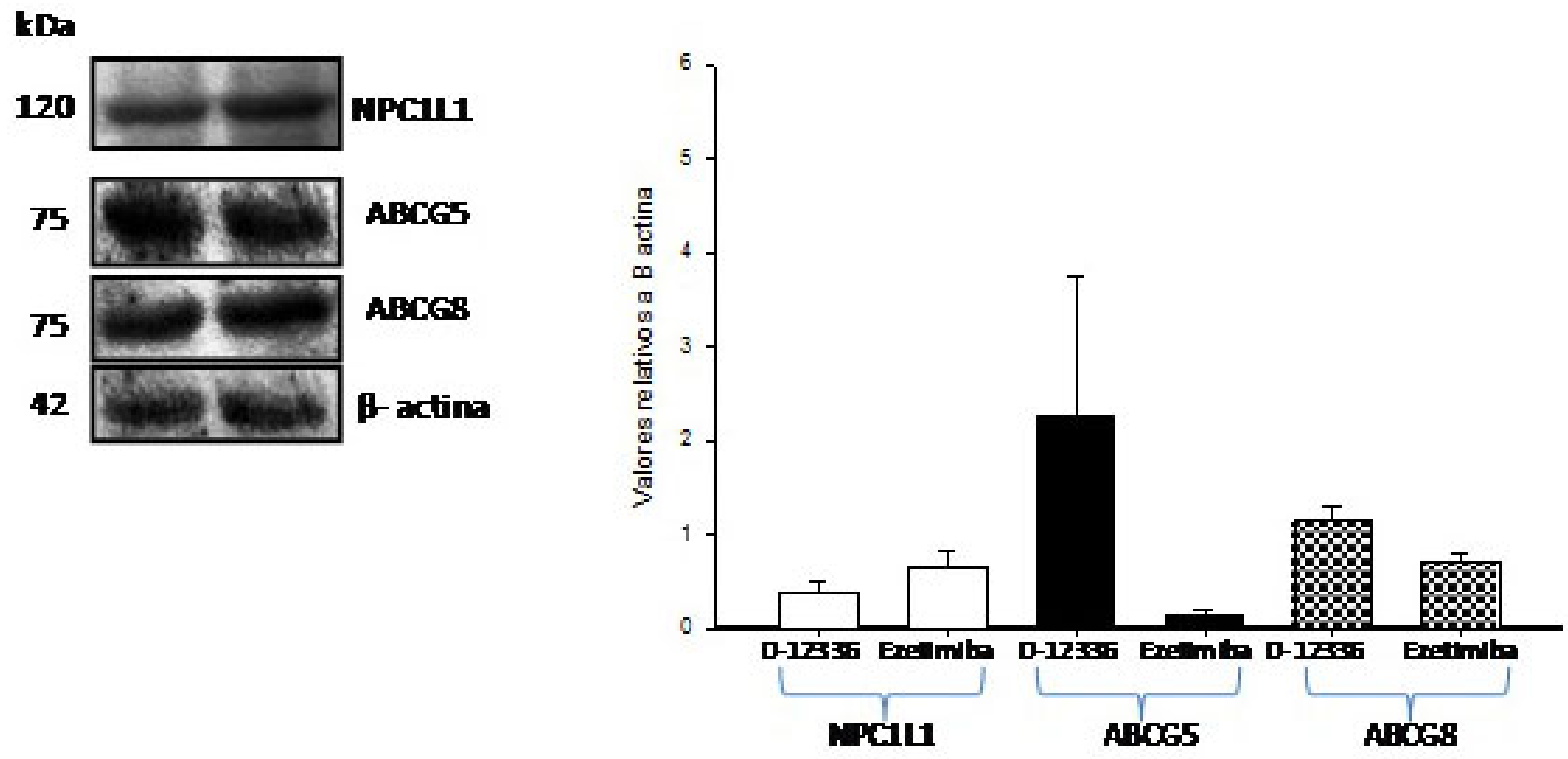

Fig. 3. Niveles de expresión de las proteínas NPC1L1, ABCG5 y ABCG8 en células del intestino delgado de ratones C57BL/6, después de un mes de tratamiento con ezetimiba. Los valores de expresión corresponden al promedio de 5 animales. 


\section{DISCUSIÓN}

Efecto de la inducción de hipercolesterolemia en la expresión relativa de los genes NPC1L1, ABCG5 y ABCG8 en células del intestino delgado de ratones C57BL/6. Los animales de este estudio fueron alimentados con la dieta D12336, que contenía $15 \%$ de grasa, $1,25 \%$ de colesterol y $0,5 \%$ de colato de sodio, esto corresponde a una dieta aterogénica, similar a la descrita por Paigen et al. Como resultado de su consumo los ratones C57BL/6 desarrollaron hipercolesterolemia, incrementando el valor de colesterol total plasmático de 1,85 $\pm 0,49 \mathrm{mmol} / \mathrm{L}$ al inicio del estudio, a 3,11 $\pm 0,73 \mathrm{mmol} / \mathrm{L}$ a las 4 semanas. Por otra parte, la concentración plasmática de triglicéridos no se afectó significativamente $(0,73 \pm 0,30$ vs. $1,08 \pm 0,50 \mathrm{mmol} / \mathrm{L}, \mathrm{P}=$ $0,221)$. Esto puede ser explicado por la presencia en la dieta de colato de sodio, componente que actúa acentuando la hipercolesterolemia inducida por el colesterol y el alto contenido graso, facilitando la absorción de grasa y colesterol, y probablemente, por la inhibición de la colesterol 7a hidroxilasa, enzima limitante en la conversión del colesterol a ácidos biliares. Además, el colato es un ligando del receptor nuclear farnesoide (FXR), cuya actividad regula la expresión de múltiples genes implicados en el metabolismo de las lipoproteínas, entre los que se cuentan los genes de las apolipoproteínas CII y CIII (Getz \& Reardon, 2006). La alteración en la expresión de estos genes podría ser la responsable del bajo contenido plasmático de triglicéridos encontrado en los animales alimentados con esta dieta.

Hasta el momento, el patrón de expresión de los genes implicados en la regulación de la absorción de colesterol tales como: NPC1L1, ABCG5 y ABCG8, no ha sido completamente establecido. En este estudio, la alimentación de los ratones con la dieta D12336, provocó un incremento de 5,4 veces en la expresión del gen NPC1L1 en relación al valor basal. Este incremento fue confirmado, al evaluar la expresión proteica de NPC1L1 mediante Western blot. Sin embargo, este resultado difiere con lo descrito por otros autores; Davis et al. (2004), concluyeron que la expresión intestinal de NPC1L1 fue reprimida en ratones tipo silvestre y NPC1L1 (+/-) alimentados con una dieta compuesta por un $1 \%$ de colesterol y $0,5 \%$ de colato de sodio. NPC1L1 posee elementos sensibles a esterol en su región promotora, lo que sugiere que su expresión es regulada por proteínas implicadas en el metabolismo del colesterol. De tal manera, la represión de NPC1L1 en presencia de una dieta alta en colesterol y colato podría deberse a la retención del complejo SREBP-SCAP en la membrana del Retículo Endoplasmico, previniendo la entrada del SREBP activo en el núcleo y evitando su unión a los elementos de respuesta sensibles a esterol en la región promotora de NPC1L1. En otro estudio, Repa et al. (2004) alimentaron ratones ACAT2 deficientes y tipo silvestre con una dieta que contenía concentraciones crecientes de colesterol hasta el 0,5\%, comprobando que la expresión de NPC1L1 fue baja en el intestino de los ratones tipo silvestre y en los ACAT2 deficientes, fue aún más baja. Field et al. (2004) experimentando con hámsteres, mostraron que al adicionar colesterol a una dieta estándar durante 14 días no se alteró la expresión de NPC1L1, mientras que la de ABCG5 y ABCG8 se incrementó. Lally et al. en ratas Sprague - Dawley, a las que se indujo diabetes mediante estreptozotocina, investigaron el efecto de una dieta estándar de laboratorio a la que se adicionó un $40 \%$ de grasa y $0,5 \%$ de colesterol, en la expresión de NPC1L1, ABCG5 y ABCG8, demostrando un incremento neto en la absorción de colesterol a través de la sobrerregulación de NPC1L1 y una represión de ABCG5 y G8 en el hígado e intestino.

Duan et al. (2006), estudiaron el efecto de la edad en la absorción de colesterol en ratones C57L/J (caracterizados por su alta absorción de colesterol) y ratones AKR/J (caracterizados por absorber poco colesterol). Los animales fueron mantenidos consumiendo una dieta estándar de laboratorio y estudiados a las 8 semanas (adultos jóvenes), 36 semanas (adultos mayores) y 50 semanas (ancianos) de edad, respectivamente. Los autores concluyeron que a medida que aumenta la edad se incrementa la absorción de colesterol, esto se vio reflejado por una reducción en la expresión de ABCG5 y ABCG8, y un aumento en la expresión de NPC1L1. En nuestro estudio, los animales fueron ingresados al protocolo después de un periodo de adaptación de 15 días, es decir con 10 semanas de vida. El efecto de la dieta hipercolesterolémica fue evaluado a las 14 semanas, constatándose un aumento en la expresión de NPC1L1, sin alteración significativa en ABCG5 y ABCG8. Es posible que el envejecimiento natural de los animales en el transcurso del estudio pueda influir, al menos en parte, en nuestros resultados.

El efecto del colesterol dietario en la expresión de ABCG5 y ABCG8 a nivel intestinal es controversial. El presente estudio muestra que la inducción de hipercolesterolemia no generó cambios significativos en el patrón de expresión génica de ABCG5 y ABCG8. Sin embargo, al evaluar la expresión proteica se aprecia un incremento en los niveles de la proteína ABCG5. Algunos estudios han demostrado que la suplementación dietética de colesterol incrementa la expresión de ABCG5 y ABCG8 en el intestino delgado; Berge et al. (2000) en ratones alimentados con una dieta con $2 \%$ de colesterol, vieron 
incrementarse el nivel de ARNm de ABCG5 y G8 2 veces después de 7 días de iniciado el experimento. Similarmente, Repa et al. (2002), reportaron un aumento en la expresión intestinal de ABCA1, ABCG5 y ABCG8 en ratones alimentados con una dieta con $1 \%$ de colesterol. Análogamente, Duan et al. (2004), demostraron la elevación del ARNm de ABCG5 y G8 en ratones bajo una dieta rica en colesterol, indicando que estos transportadores cumplen un importante rol en la regulación de la absorción intestinal de colesterol. Por el contrario, el estudio de Davis et al. (2004), no mostró una diferencia significativa en la expresión de ABCG5 o ABCG8 al someter a ratones NPC1L1 (-/-) a una dieta rica en colesterol. Asimismo, Plösch et al. (2006), demostraron que la expresión de ABCG5 y ABCG8 no se altera al inducir la reducción de colesterol mediante la suplementación de una dieta alta en colesterol con esteroles de plantas y estanol. En ratones alimentados con una dieta libre de colesterol - alta en grasa, se demostró que los genes NPC1L1, ABCA1, ABCG5 y ABCG8 fueron reprimidos después de 2, 4 y 8 semanas de dieta, concluyéndose que los ácidos grasos no saturados son los responsables de la represión de los genes encargados del transporte de colesterol. La ingesta de la dieta alta en grasa provoca una disminución en la absorción de colesterol. Según los autores, es posible que con el objeto de liberar colesterol intracelular para la formación de quilomicrones, el eflujo de colesterol a través de los transportadores $\mathrm{ABC}$ sea reducido. Además, para compensar la disminución en la captación de colesterol en el enterocito, la síntesis de colesterol es inducida.

El mecanismo por el cual los ácidos grasos ejercen su efecto aun no está esclarecido. Es conocido que la expresión de NPC1L1 se encuentra regulada por receptores nucleares, principalmente por LXR, sin embargo, se ha demostrado que en una dieta alta en grasa, la represión de NPC1L1 no depende de LXRa, esto ha llevado a que surjan otros candidatos como los receptores activados por proliferadores de peroxisomas (PPARs) que podrían cumplir este rol (de Vogel-van den Bosch et al., 2008). Es posible que en nuestro estudio el alto contenido graso de la dieta (15\%), influya también en la reducción de la expresión de los transportadores ABCG5 y G8, sin embargo, el efecto podría ser compensado por la presencia de una dosis alta de colesterol en la fórmula ( $1,25 \%)$, resultando finalmente, en que la expresión proteica de ABCG5 se vea aumentada.

Bloks et al. (2004), empleando un modelo de ratas Wistar con diabetes inducida por estreptozotocina, evaluaron la expresión de los genes ABCG5 y G8 a nivel hepático e intestinal, concluyendo que la expresión hepática se redujo para ambos genes como consecuencia del desarrollo de diabetes. A nivel intestinal se observó una reducción similar, la que fue confirmada por la disminución en la expresión proteica.
Efecto del tratamiento con ezetimiba en la expresión relativa de los genes NPC1L1, ABCG5 y ABCG8 en células del intestino delgado de ratones C57BL/6. En los años recientes, uno de los avances más significativos en la terapia hipolipemiante ha sido el desarrollo del inhibidor de la absorción intestinal de colesterol; ezetimiba. En el presente estudio, a un grupo de los animales hipercolesterolémicos $(\mathrm{n}=5)$ se les administró una dosis de $5 \mathrm{mg} / \mathrm{Kg} /$ día de ezetimiba, lo que provocó una reducción significativa en el valor de colesterol total de $3,11 \pm 0,73$ a $2,11 \pm 0,50 \mathrm{mmol} /$ $\mathrm{L}(\mathrm{P}=0,002)$. Este resultado se explica porque la ezetimiba actúa inhibiendo selectivamente el transporte de colesterol a través de la pared intestinal sin afectar la hidrólisis intestinal de esteres de colesterol ni la absorción de los ácidos grasos aquí generados. El colesterol liberado producto de esta hidrólisis no es absorbido en presencia de ezetimiba (Davis \& Veltri, 2007). En nuestro estudio, el tratamiento de los animales con ezetimiba no afectó significativamente los niveles séricos de triglicéridos $(\mathrm{P}=0,162)$. Estudios previos han establecido que la ezetimiba no ejerce efecto sobre los valores de triglicéridos plasmáticos (Davis \& Veltri). Nuestros resultados claramente reafirman esta idea, sin embargo, recientemente se ha sugerido que la inhibición de la absorción de colesterol puede impactar en la absorción de las grasas de la dieta y/o en su metabolismo y proteger frente a la obesidad inducida por dieta. En algunos modelos animales alimentados con dietas altas en grasa y colesterol, la ezetimiba ha reducido los valores de triglicéridos plasmáticos, sugiriendo que alguna vía sensible a ezetimiba pueda modular el metabolismo de las grasas. En este sentido, Labonté et al. (2008) han planteado que el tratamiento con ezetimiba o la falta de la proteína NPC1L1, reducen la absorción de ácidos grasos saturados por un mecanismo que puede involucrar una expresión reducida de la proteína transportadora de ácidos grasos (FATP4).

En los ratones C57BL/6 tratados con ezetimiba, la expresión génica de NPC1L1 no fue significativamente diferente de la que se presentaba en el estado basal. Este estudio mostró, además, que después del tratamiento con ezetimiba se incrementó el nivel de ARNm de ABCG5. En el caso del gen ABCG8, se aprecia una tendencia a la sobreexpresión que no es significativa. Por otro lado, al evaluar la expresión proteica se observó un aumento de NPC1L1 y una reducción de ABCG5 y ABCG8 a nivel intestinal. Es probable que la inhibición de la absorción de colesterol mediada por ezetimiba, produzca un estado de depleción de colesterol al interior del enterocito, lo que llevaría a que se reprima la expresión proteica de ABCG5 y ABCG8 evitando así el eflujo de colesterol. Además, como mecanismo compensatorio, la expresión de NPC1L1, ABCG5 y ABCG8 podría ser inducida. Se cree que ezetimiba impide la absorción de colesterol bloqueando la endocitosis de NPC1L1, 
esto porque competiría con el colesterol para unirse al dominio sensible a esterol presente en NPC1L1. Nuestros datos muestran que la expresión génica de NPC1L1 no varía significativamente con la administración de ezetimiba, sin embargo, se advierte una mayor cantidad de proteína NPC1L1 disponible en la membrana del enterocito (Figura 3). Esta discrepancia podría deberse por una parte a que los niveles de NPC1L1 en el intestino son variables y a que los animales tengan una respuesta diferente entre sí al tratamiento. Esto fue analizado por Chan et al. (2008), al tratar zarigüeyas con una dieta alta en colesterol y ezetimiba comprobando diferencias en la expresión intestinal de NPC1L1 entre respondedores y no respondedores. Además, en cerdos tratados con ezetimiba, se ha demostrado la sobreexpresión intestinal y hepática de NPC1L1, la que estaría ocasionada por una depleción de colesterol en ambos tejidos. Esto también indica la diferente respuesta que se presenta dependiendo del modelo animal estudiado (Telford et al., 2006). En un estudio efectuado en hámsteres, se evaluó la expresión de los genes NPC1L1, ABCG5 y ABCG8 como resultado de la administración de una dieta rica en colesterol y grasa, y posterior tratamiento con ezetimiba. Aquí se apreció que la expresión de NPC1L1 no experimentó cambios como resultado del tratamiento. Por el contrario, los niveles de ARNm de ABCG5 y ABCG8 cayeron significativamente en respuesta al tratamiento, retornando rápidamente a la normalidad una vez que fue cesada la administración de ezetimiba. En este estudio, además se constató un incremento en la expresión de la HMG- CoA reductasa como mecanismo compensatorio ante la disminución de colesterol (Valasek et al., 2008).

Finalmente, nuestros resultados sugieren que tanto la dieta hipercolesterolémica como el tratamiento con ezetimiba en un modelo experimental, afectan los niveles de expresión de NPC1L1, ABCG5 y ABCG8, indicando que ABCG5 y ABCG8 están involucrados en la respuesta a este fármaco. No obstante, el mecanismo mediante el cual se explica esta interacción debe ser investigado en un futuro estudio.

CAAMAÑO, J.M.; SAAVEDRA, N.; WULFF, C. \& SALAZAR, L.A. Therapeutic response to ezetimibe in C57BL/6 mice is mediated by changes in NPC1L1, ABCG5 and ABCG8 expression in the enterocyte. Int. J. Morphol., 30(2):531-540, 2012.

SUMMARY: Proteins NPC1L1, ABCG5 and ABCG8 are involved in the intestinal absorption of cholesterol. Ezetimibe inhibits this process by blocking NPC1L1, however, its effect on ABCG5 and ABCG8 is not yet clear. Thus, the objective of this study was to evaluate in C57BL / 6 mice with diet-induced hypercholesterolemia treated with ezetimibe, the expression of NPC1L1, ABCG5 and ABCG8 by real time PCR and Western blot, in 3 groups of animals: 1, diet hypercholesterolemic D12336, 2, D12336 diet plus 5 mg/kg/ day of ezetimibe, 3 , diet control. The serum level of total cholesterol was significantly different between groups (control: $1.85 \pm 0.49$ $\mathrm{mmol} / \mathrm{L}$; diet D12336: $3.11 \pm 0.73 \mathrm{mmol} / \mathrm{L}$; ezetimibe: $2.11 \pm 0.50 \mathrm{mmol} / \mathrm{L}, \mathrm{P}=0.001)$. NPC1L1 gene expression increased 5.4-fold in the group receiving the diet $\mathrm{D} 12336(\mathrm{P}=0.003)$. Furthermore, the gene expression of ABCG5 and ABCG8 was not different in the group with hypercholesterolemia $(\mathrm{P}=0.239$ and $\mathrm{P}=0.201$, respectively). After treatment with ezetimibe, $\mathrm{ABCG}$ gene expression was increased 15.6 times $(\mathrm{P}=0.038)$. No significant differences in gene expression of NPC1L1 $(\mathrm{P}=0.134)$ and $\mathrm{ABCG} 8(\mathrm{P}=0.067)$. Regarding protein expression, the D12336 diet increased the levels of expression of NPC1L1 $(\mathrm{P}=0.022)$ and $\mathrm{ABCG} 5(\mathrm{P}=0.008)$, treatment with ezetimibe increased the levels of NPC1L1 $(\mathrm{P}=0.048)$ and reduced levels of ABCG5 $(\mathrm{P}=0.036)$ and $\mathrm{ABCG} 8(\mathrm{P}=0.016)$. In conclusion, our results suggest that both hypercholesterolemic diet as treatment with ezetimibe, in an experimental model, affect the expression levels of NPC1L1, ABCG5 and ABCG8, suggesting that ABCG5 and ABCG8 are involved in lipid-lowering response to this drug. However, the mechanism by which this interaction is explained requires further study.

KEY WORDS: Ezetimibe; Cholesterol; NPC1L1; ABCG5/G8.

\section{REFERENCIAS BIBLIOGRÁFICAS}

Acalovschi, M.; Ciocan, A.; Mostean, O.; Tirziu, S.; Chiorean, E.; Keppeler, H.; Schirin-Sokhan, R. \& Lammer, F. Are plasma lipid levels related to ABCG5/ABCG8 polymorphisms? A preliminary study in siblings with gallstones. Eur. J. Intern. Med., 17(7):490-4, 2006.

Altmann, S. W.; Davis, H. R. Jr.; Zhu, L. J.; Yao, X.; Hoos, L. M.; Tetzloff, G.; Iyer, S. P.; Maguire, M.; Golovko, A.; Zeng, M.; Wang, L.; Murgolo, N. \& Graziano, M. P. Niemann-Pick C1 like 1 protein is critical for intestinal cholesterol absorption. Science, 303:1201-4, 2004.
Berge, K. E.; Tian, H.; Graf, G. A.; Yu, L.; Grishin, N. V.; Schultz, J.; Kwiterovich, P.; Shan, B.; Barnes, R. \& Hobbs, H. H. Accumulation of dietary cholesterol in sitosterolemia caused by mutations in adjacent ABC transporters. Science, 290(5497):1771-5, 2000.

Bloks, V. W.; Bakker-Van Waarde, W. M.; Verkade, H. J.; Kema, I. P.; Wolters, H.; Vink, E.; Groen, A. K. \& Kuipers, F. Downregulation of hepatic and intestinal Abcg 5 and Abcg8 expression associated with altered sterol fluxes in rats with streptozotocininduced diabetes. Diabetologia, 47(1):104-12, 2004. 
Boehm, O.; Zur, B.; Koch, A.; Tran, N.; Freyenhagen, R.; Hartmann, M. \& Zacharowski, K. Clinical chemistry reference database for Wistar rats and C57/BL6 mice. Biol. Chem, 388:547-54, 2007.

Chan, J.; Kushwaha, R. S.; Vandeberg, J. F. \& Vandeberg, J. L. Effect of ezetimibe on plasma cholesterol levels, cholesterol absorption, and secretion of biliary cholesterol in laboratory opossums with high and low responses to dietary cholesterol. Metabolism, 57(12):1645-54, 2008.

Davis, H. R. Jr.; Zhu, L. J.; Hoos, L. M.; Tetzloff, G.; Maguire, M.; Liu, J.; Yao, X.; Iyer, S. P.; Lam, M. H.; Lund, E. G.; Detmers, P. A.; Graziano, M. P. \& Altmann, S. W. Niemann-Pick C1 Like 1 (NPC1L1) is the intestinal phytosterol and cholesterol transporter and a key modulator of whole-body cholesterol homeostasis. J. Biol. Chem., 279(32):33586-92, 2004.

Davis, H. R. \& Veltri, E. P. Zetia: inhibition of Niemann-Pick C1 like 1 (NPC1L1) to reduce intestinal cholesterol absorption and treat hyperlipidemia. J. Atheroscler. Thromb., 14(3):99108, 2007.

de Vogel-van den Bosch, H. M.; de Wit, N. J.; Hooiveld, G. J.; Vermeulen, H.; van der Veen, J. N.; Houten, S. M.; Kuipers, F.; Müller, M. \& van der Meer, R. A cholesterol-free, high-fat diet suppresses gene expression of cholesterol transporters in murine small intestine. Am. J. Physiol. Gastrointest. Liver Physiol., 294(5):G1171-80, 2008.

Duan, L. P.; Wang, H. H. \& Wang, D. Q. Cholesterol absorption is mainly regulated by the jejunal and ileal ATP-binding cassette sterol efflux transporters Abcg5 and Abcg8 in mice. J. Lipid Res., 45:1312-23, 2004.

Duan, L. P.; Wang, H. H.; Ohashi, A. \& Wang, D. Q. Role of intestinal sterol transporters Abcg5, Abcg8, and Npc111 in cholesterol absorption in mice: gender and age effects. Am. J. Physiol. Gastrointest. Liver Physiol., 290(2):G269-76, 2006.

Field, F. J.; Born, E. \& Mathur, S. N. Stanol esters decrease plasma cholesterol independently of intestinal $\mathrm{ABC}$ sterol transporters and Niemann-Pick C1-like 1 protein gene expression. J. Lipid Res, 45(12):2252-9, 2004.

Fossati, P. \& Medicci, R. Abstract Book. International Symposium on Cholesterol Control and Cardiovascular Diseases: Prevention and Therapy. Milan Italy, 1987. Apud: Bayer Corporation, Diagnostic Division, Tarrytown, N.Y., Cholesterol-Fast color.

Fossati, P. \& Prencipe, L. Serum triglycerides determined colorimetrically with an enzyme of low-density lipoprotein cholesterol in plasma without use of the preparative ultracentrifuge. Clin. Chem., 28:2077-80, 1982.

Garcia-Calvo, M.; Lisnock, J.; Bull, H.; Hawea, B.; Burnett, D.; Braun, M.; Crona, J.; Davis, H.; Dean, D.; Detmers, P.;
Graziano, M.; Hughes, M.; Macintyre, E.; Ogawa, A.; O'neill, K.; Iyer, S.; Shevell, D.; Shmith, M.; Tang, Y.; Makarewicz, A; Ujjainwalla, F.; Altmann, S.; Chapman, K. \& Thornberry, N. The target of ezetimibe is Niemann-Pick C1-like (NCP1L1). Proc. Natl. Acad. Sci. USA, 102:8132-37, 2005.

Ge, L.; Wang, J.; Qi, W.; Miao, H. H.; Cao, J.; Qu, Y. X.; Li, B. L. \& Song, B. L. The cholesterol absorption inhibitor ezetimibe acts by blocking the sterol-induced internalization of NPC1L1. Cell Metab., 7(6):508-19, 2008.

Getz, G. S. \& Reardon, C. A. Diet and murine atherosclerosis. Arterioscler. Thromb. Vasc. Biol., 26(2):242-9, 2006.

Hui, D. \& Howles, P. Molecular mechanisms of cholesterol absorption and transport in the intestine. Semin. Cell Dev. Biol., 16:183-92, 2005.

Klett, E. L. \& Patel, S. B. Will the real cholesterol transporter please stand up. Science, 303(5661):1149-50, 2004.

Kosoglou, T.; Statkevich, P.; Johnson-Levonas, A., Paolini, J.; Bergman, A. \& Alton, K. Ezetimibe. A review of its metabolism, pharmacokinetics and drug interactions. Clin. Pharmacokinet., 44:457-94, 2005.

Labonté, E. D.; Camarota, L. M.; Rojas, J. C.; Jandacek, R. J.; Gilham, D. E.; Davies, J. P.; Ioannou, Y. A.; Tso, P.; Hui, D. Y. $\&$ Howles, P. N. Reduced absorption of saturated fatty acids and resistance to diet-induced obesity and diabetes by ezetimibe-treated and Npc111-/- mice. Am. J. Physiol. Gastrointest. Liver Physiol., 295(4):G776-83, 2008.

Lally, S.; Tan, C.; Owens, D. \& Tomkin, G. Messenger RNA levels of genes involved in dysregulation of postprandial lipoproteins in type 2 diabetes: the role of Niemann-Pick C1 like 1, ATPbinding cassette, transporters G5 and G8, and microsomal triglyceride transfer protein. Diabetologia, 49:1008-16, 2006.

Lammert, F. \& Wang, D. New insights into the genetic regulation of intestinal cholesterol absorption. Gastroenterology, 129:718$34,2005$.

Livak, K. \& Schmittgen, T. Analysis of relative gene expression data using real-time quantitative PCR and the 2(-Delta Delta C(T)) Method. Methods, 25:402-08, 2001.

Oswald, S.; Westrup, S.; Grube, M.; Kroemer, H. K.; Weitschies, W. \& Siegmund, W. Disposition and sterol-lowering effect of ezetimibe in multidrug resistance associated protein 2-deficient rats. J. Pharmacol. Exp. Ther., 318(3):1293-99, 2006.

Paigen, B.; Morrow, A.; Brandon, C.; Mitchell, D. \& Holmes, P. Variation in susceptibility to atherosclerosis among inbred strains of mice. Atherosclerosis, 57(1):65-73, 1985.

Plösch, T.; Kruit, J. K.; Bloks, V. W.; Huijkman, N. C.; Havinga, R.; Duchateau, G. S.; Lin, Y. \& Kuipers, F. Reduction of 
CAAMAÑO, J.M.; SAAVEDRA, N.; WULFF, C. \& SALAZAR, L.A. La respuesta terapéutica a ezetimiba en ratones C57BL/6 es mediada por cambios en la expresión de NPC1L1, ABCG5 y ABCG8 en el enterocito. Int. J. Morphol., 30(2):531-540, 2012.

cholesterol absorption by dietary plant sterols and stanols in mice is independent of the Abcg5/8 transporter. J. Nutr., 136(8):2135-40, 2006.

Repa, J. J., Buhman, K. K.; Farese, R. V. Jr.; Dietschy, J. M. \& Turley, S. D. ACAT2 deficiency limits cholesterol absorption in the cholesterol-fed mouse: impact on hepatic cholesterol homeostasis. Hepatology, 40(5):1088-97, 2004.

Repa, J. J.; Berge, K. E.; Pomajzi, C.; Richardon, J. A.; Hobbs, H. \& Mangelsdorf, D. J. Regulation of ATP-binding cassette sterol transporters ABCG5 y ABCG8 by the liver X receptors a and b. J. Biol. Chem., 277:18793-800, 2002.

Schmitz, G.; Langmann, T. \& Heimerl, S. Role of ABCG1 and other ABCG family members in lipid metabolism. J. Lipid Res., 42:1513-20, 2001

Telford, D. E.; Sutherland, B. G.; Edwards, J. Y.; Andrews, J. D.; Barrett, P. H. R. \& Huff, M. W. Ezetimibe plus simvastatin decreases LDL apolipoprotein $\mathrm{B}$ via enhanced clearance mediated by increased hepatic expression of LDL receptors. Atherosclerosis supplements, 7: 482, 2006.

Temel, R. E. \& Rudel, L. L. Diet effects on atherosclerosis in mice. Curr. Drug Targets, 8(11):1150-60, 2007.

Valasek, M. A.; Repa, J. J.; Quan, G.; Dietschy, J. M. \& Turley, S. D. Inhibiting intestinal NPC1L1 activity prevents diet-induced increase in biliary cholesterol in Golden Syrian hamsters. Am. J. Physiol. Gastrointest. Liver Physiol., 295(4):G813-22, 2008.

\author{
Dirección para correspondencia: \\ Prof. Dr. Luis Antonio Salazar \\ Departamento de Ciencias Básicas, \\ Facultad de Medicina - Universidad de La Frontera \\ Av. Francisco Salazar 01145. Casilla 54-D \\ Temuco, \\ CHILE \\ Tel.: +56 45592 895; \\ Fax: +56 45592832 \\ E-mail: Isalazar@ufro.cl
}

Recibido : 22-02-2012

Aceptado: 18-03-2012 